\title{
Systemic Inflammation in Psoriasis Vulgaris (Study of hs-CRP and alfa-TNF in Albanian Psoriatic Patients)
}

\author{
Migena Vargu ${ }^{1}$, Petrit Vargu ${ }^{2}$, Nevila Heta ${ }^{3}$, Eliverta Kondi ${ }^{4}$, Mimoza Lezha $^{5}$ \\ ${ }^{1}$ Dermato-Venerologic Clinic, U niversity Hospital Center :"Mother Teresa” Tirana, Albania \\ ${ }^{2,4,5}$ Cardiology Clinic, University Hospital Center :"Mother Teresa" Tirana, Albania \\ ${ }^{3}$ Clinic Laboratory, University Hospital Center :"Mother Teresa" Tirana, Albania
}

\begin{abstract}
Studies investigating systemic inflammation in psoriasis use different serum markers and report discrepant results. We set out to determine whether systemic inflammation is elevated in patients with psoriasis compared with healthy controls, and to measure the extent of this elevation, by summarizing available data on serum inflammatory markers.
\end{abstract}

Keywords: psoriasis, inflammation, hs-CRP, alpha-TNF

\section{Psoriasis Pathogenesis}

Continuous studies in the domain of molecular biology and immunology indicate psoriasis as a disease with very complex characteristics with respect to its pathogenesis. A large number of inflammatory cells are included in its pathogenesis where a major role is being played by lymphocites- $\mathrm{T}$, cytokines and chemiokines.

Gottleib together with his collaborators showed in 1995 that psoriasis could be successfully treated by means of toxine lymphocyte selective DAB389-IL-2, a discovery which set the basis of a new era as far as it concerns the psoriasis treatment through immunology target inhibitors. 1

The vast infiltrate with mononuclear immune cells, in derma and epiderma ( $\mathrm{T}$ cells and the dentric ones in derma as well as polymorphonuclear leucocytes like for instance neutrophyles at stratum comeun) are very important elements from the histological point of view as well as for the psoriasis pathogenesis. It is considered that psoriasis pathogenesis is a immunologic process which needs a genetic basis. From this consideration, the presence of a still unknown (auto) antigene influences in $\mathrm{T}$ cells excitement, which then infiltrate the skin and start the inflammatory process. Hence, it is exactly this coordination between external factors (physical trauma or bacterial infections) and genetic ones which activate the inflammatory process. $2-4$

It is believed that lymphocytes play a key role in psoriasis pathogenesis, but particulary lymphocytes Thl (T-helper) are being identified as the main source of inflammatory cytokines production which take part in the disease pathogenesis.5

Keratinocytes contribute in the cutaneus immune response by means of cytokine manifestation and especially of IL-23. The latter activate lymphocyte $-\mathrm{T}$ (memory), which release cytokine type 1 like for instance interferon -alfa (IFN-alfa ,tumoral necrosis factor- $\alpha(\mathrm{TNF}-\alpha)$ and IL-2, which play a role in the cascade inflammatory process .6 .7

\section{CRP Role}

In 1930, Tillet and Francis observed a substance in the serum of individuals with pneumococcal infection, which left a precipitation during the stirring with the C-polisaccaride coverage of streptococcus pneumoniae. They noticed that this C-reactive activity was missing in healthy individuals. Furthermore, MacLeod and Avery( 9), describes this substance as a protein of the acute phase. Right after it, Lofsrom (10) proved the presence of the acute phase response (ARP), both in acute and chronic infections. Step by step, CRP became known as a non specific protein of the acute phase.

The latest data show that, CRP is not just a inflammatory marker but it plays an active role in atherogenesis. CRP stimulates the release of inflammatory cytokines from monocytes like for instance: IL $1 \beta$, IL 6 , TNF $\alpha$; increases ICAM1 and VCAM1 manifestation from endothelial cells; Intermediates the MCP1 action in endothelial cells (an effect that is stopped by use of simvastinate and fenofibers); agregates LDL and IDL in early atheschlerotic plaques.

\section{3. hs- CRP Dosage Methods}

In lab practice there are being used, nowadays, two types of CRP dosing. This derives from the fact that CRP does exist normally in plasma at very low concentrations which are independent from the acute infections but they could change during cardiovascular diseases which are being associated with a low degree chronic inflammation. In cases of acute 


\section{International Journal of Science and Research (IJSR) \\ ISSN (Online): 2319-7064 \\ Index Copernicus Value (2013): 6.14 | Impact Factor (2014): 5.611}

inflammations of high degree, which are being developed in traumas, surgeries, bacterial infections and arthritis the CRP levels are being measured with the common lab methods since these situations cause a very high CRP concentration. Usually, acute inflammations are being associated with CRP increase values of higher than $10 \mathrm{mg} / \mathrm{L}$, while the chronic inflammation in low degree caused by atherosclerosis is associated with $\mathrm{CRP}$ values lower than $10 \mathrm{mg} / \mathrm{L}$. Slight inflammations and viral infection in general, cause an increase of CRP concentration from $10-50 \mathrm{mg} / \mathrm{l}$, while the severe and active ones or even the bacterial infections, can cause an increase of $50-200 \mathrm{mg} / \mathrm{l}$.

The standard clinical methods for CRP dosage have a detection limit of $3-8 \mathrm{mg} / \mathrm{L}$. Therefore these methods lose their sensitivity in lower normal level and cannot be used effectively for the vascular risk prevention. As a consequence, nowadays several methods called "high-sensitivity" or "ultra-sensitive" are being processed and implemented and moreover standardizing programs are being taken to ensure comparability among hsCRP dosage methods. So today it is exactly the hs-CRP dosage method which covers the values $0.1-10 \mathrm{mg} / \mathrm{L}$, this method which provides us a far better assessment of a chronic and latent inflammation.

Even in many psoriasis patients it has been noticed an increased level of hs-CRP. They tend to drop during treatment but usually do not turn to normal values. As far as it concerns the healthy population, there are today many prospective papers showing that CRP levels are an independent risk factor of the atherosclerotic process and at the same time a prediction of the future cardiovascular events.10. These papers have included sexes, European and non-European, and all ages. In addition, CRP has been evaluated even as a predictor of cerebral shocks and peripheral arterial diseases.11

Hs-CRP dosing is not valid in patients with confirmed inflammatory processes, as in many studies about $2 \%$ of patients had CRP values above $2 \mathrm{mg} / \mathrm{dl}$, values that are usually associated with other inflammatory processes.

Hence, the use of an easily measurable systemic inflammatory marker such as hs-CRP, with a relatively long half-life, $19 \mathrm{hrs}$ and independent of circadian pace, can determine which patients are at higher risk of atherosclerotic and who among those with coronary disease have higher risk to develop future cardiovascular events. 12

\section{TNF-alfa}

Genetic studies indicate that psoriasis and cardiovascular diseases manifest common mechanisms, eg inflammatory cytokines, TNF-alpha and IL-1 which play a role in chronic inflammation of psoriasis which in turn promotes a risky cardiovascular profile. (Mentioned above in the pathogenesis of psoriasis and cardiovascular diseases) . TNF-alpha itself is one of the cytokines involved in acute coronary syndromes. TNF-alpha is found at high levels in the blood and skin of psoriasis patients.

\section{Volume 4 Issue 11, November 2015}

The search for markers in psoriasis was revived, as not only were these found in the skin, but researchers also identified a spillover of inflammatory markers into the systemic circulation, using them to measure disease severity, to monitor treatment response objectively, to find new targets for therapy and to explain comorbidities in patients with psoriasis. 13

\section{Material and Method}

There have been studied 2 groups.

Psoriasis group patients (number of studied cases 199). In this group were included patients of both sexes hospitalized in the clinic of Dermatology-Venerology in University Hospital Center "Mother Teresa" . Psorias diagnose is a clinical one. Only in doubtful cases (differential diagnosis with Pytiriasis Rosea or Ecsema psoriasiforme) it was taken biopsy examination that confirmed psoriasis diagnosis. There were excluded from this group all individuals aged less than 18 years, having a current/or past history of acute myocardial infarct, peripheral artery disease or coronary arteries, or known presence of inflammatory diseases. It was calculated PASI for all the patients and for how many years they have psoriasis. Control group of non psoriasis individuals (number of cases taken into the study was 199). The excluding criteria from the study were the same as in the first group. Samples have been taken for analyzing hs -CRP and TNF-alpha.

PASI calculation method

a) Body is divided in four areas: Head, arms, body and legs.

b) It is conducted a scoring for erythema, squam, infiltration of each area (from: 0-4).

c) Scores are being added for erythema, squame and infiltration of each area.

d) Percentage of the influenced surface according to areas is being converted in scores

$(0=0 \% ; 1=<10 \% ; 2=10-<30 \% ; 3=30-<50 \% ; 4=50-<70 \%$; $5=70-<90 \% ; 6=90-<100 \%$ ).

e) (c) value is multiplied with (d) according to the areas with $0.1,0.2,0.3,0.4$, for head, arms, body and legs respectively.

The accomplishment of the laboratory part of the study is being carried out at Biochemical lab of University Hospital Center "Mother Teresa".

\section{Blood Sample Processing}

Blood has been collected in morning with empty stomach, in 5 $\mathrm{ml}$ without anticoagulants for the dosage of the specific study parameters.

The exploration of specific study parameters like for instance hs- CRP, TNF-alfa is been carried out with the autoanalyzer model DPC, IMMULITE 1000. For patient involved in this study, it has been collected their blood which was preserved in frozen state ( -80 degrees Celsius) to be analyzed in a second moment. 


\section{International Journal of Science and Research (IJSR) \\ ISSN (Online): 2319-7064 \\ Index Copernicus Value (2013): 6.14 | Impact Factor (2014): 5.611}

hs-CRP and TNF-alfa dosage is based upon an immunoenzymatic chemiluminiscent method, in solid state, carried out with the auto analyser DPC, IMMULITE 1000. hsCRP dosage in auto analyzer IMMULITE comprises a sample diluition method described as follows:

All samples were diluted in 1 to 101 ratio, by making use of CRP diluter: $10 \mu \mathrm{L}$ serum with $1000 \mu \mathrm{L}$ diluting solution. The analyzer calculates automatically the CRP and TNF-alfa concentration value.

The reference value of method for hs-CRP is $0.1-1.1 \mathrm{mg} / \mathrm{dl}$, while the one of TNF-alpha is $0.000-8.10 \mathrm{pg} / \mathrm{ml}$. The adjustment process has been carried out prior to each dosage cycle.

\section{Statistical Analysis}

The following have used for the data analysis:

Spearman correlation coefficients (for non-parametricordinary data) were applied for the evaluation of the linear relationship among numerical.

The exact Fisher test was applied for the proportion comparison of category variables. In all cases, the values of $\mathrm{P}<0.05$ were considered as statistically significant (considerable). The whole statistical analysis was carried out through the Statistic Package for Social Science, version 19.0 (SPSS - Statistical Package for Social Sciences Inc., Chicago, IL, USA).

\section{Outcomes}

Comparison of hs-CRP and alfa-TNF values (in psoriasis and control group)

$99.5 \%$ of psoriasis patients have a CRP value of $>1.10 \mathrm{mg} / \mathrm{L}$ versus $57.8 \%$ in control group and this difference is statistically very significant (Fischer exact test: $\mathrm{P}<0.001$ ), e.g. CRP values $>1.10 \mathrm{mg} / \mathrm{L}$ are an independent risk factor at psoriasis group. This finding is reflected in the following graphic 1)

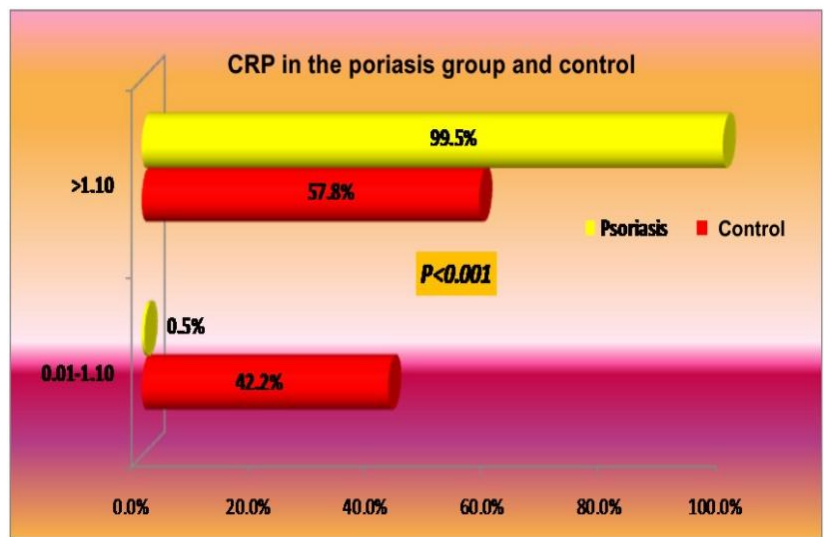

Graphic 1: Distribution of hs-CRP values in psoriasis and control group
$32.7 \%$ of non - psoriasis patients have a TNF-alpha value of $>$ $8.10 \mathrm{pg} / \mathrm{ml}$ versus $65.3 \%$ in psoriasis group and this difference was statistically highly significant (Fisher's exact test: $\mathrm{P}<0.001)$.

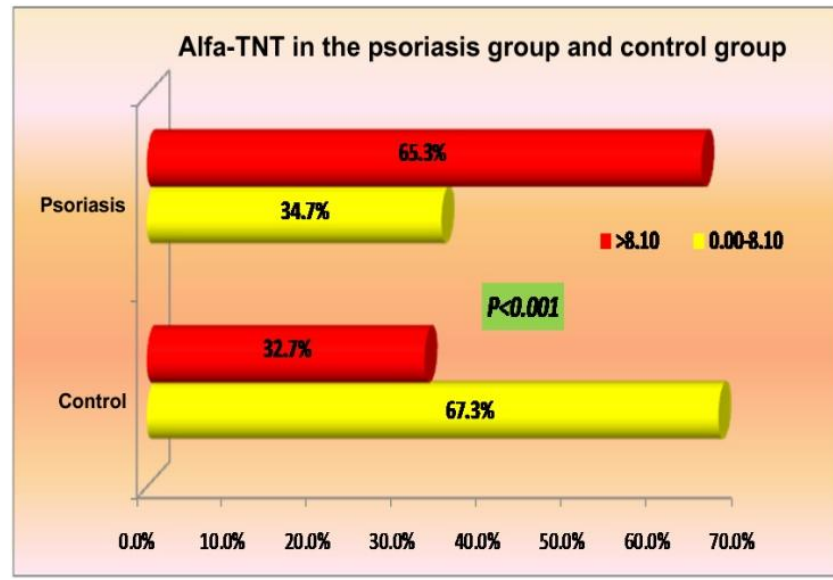

Graphic 2: Presentation of distribution of values of TNFalpha in patients with psoriasis and control group.

\section{PASI correlation with alfa-TNF and CRP}

There is a relatively strong correlation between PASI and alfaTNF: Spearman correlation coefficient $=\mathbf{0 . 5 7}$, $\mathbf{P}=\mathbf{0 . 0 3 4}$ (graphic nr 3)

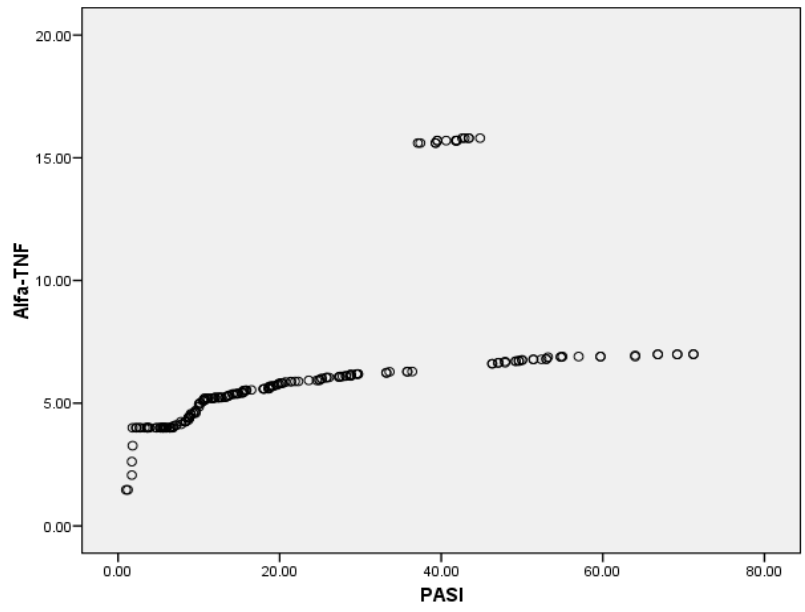

Graphic 3: Correlation between PASI and alfa-TNF

In addition, there is a relatively strong correlation between PASI and CRP: Spearman correlation coefficient $=\mathbf{0 . 4 9}$, $\mathbf{P}=\mathbf{0 . 0 4 8}$ (graphic nr .4) 


\section{International Journal of Science and Research (IJSR) \\ ISSN (Online): 2319-7064}

Index Copernicus Value (2013): 6.14 | Impact Factor (2014): 5.611

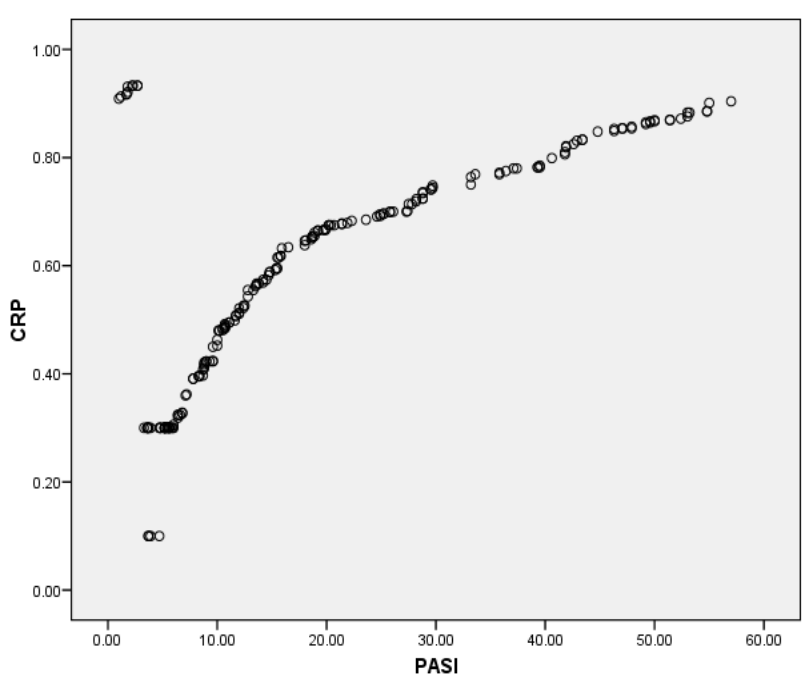

Correlation of psoriasis duration (over the years) with alpha-TNF and CRP

On the their side, there is no evidence of any significant correlation between hs-CRP and psoriasis duration (in years) in the patient group suffering from this pathology: Spearman correlation coefficient $=\mathbf{0 . 0 2 9}, \mathbf{P}=\mathbf{0 . 6 8 9}$ (graphic $\mathrm{nr} .5$ )

Graphic 4: Correlation between PASI and CRP

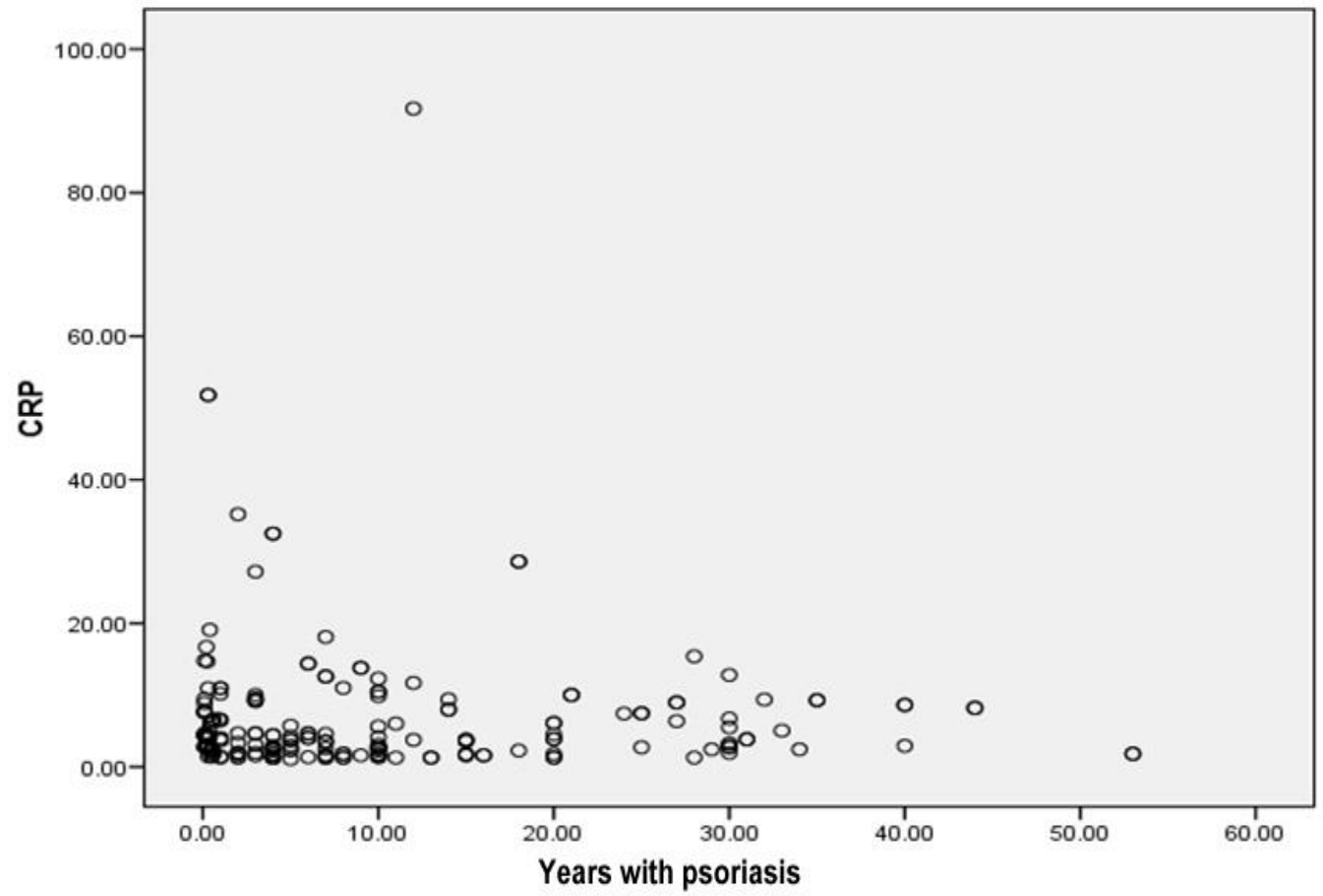

Graphic 5: correlation between hs-CRP and psoriasis duration (in years)

Moreover, there is no evidence of any significant correlation between alfa-TNF and psoriasis duration (in years) in the patient group suffering from this pathology: Spearman correlation coefficient $=\mathbf{0 . 0 7 3}, \mathbf{P}=\mathbf{0 . 3 2 5}$ (graphic nr. 6) 


\section{International Journal of Science and Research (IJSR)}

ISSN (Online): 2319-7064

Index Copernicus Value (2013): 6.14 | Impact Factor (2014): 5.611

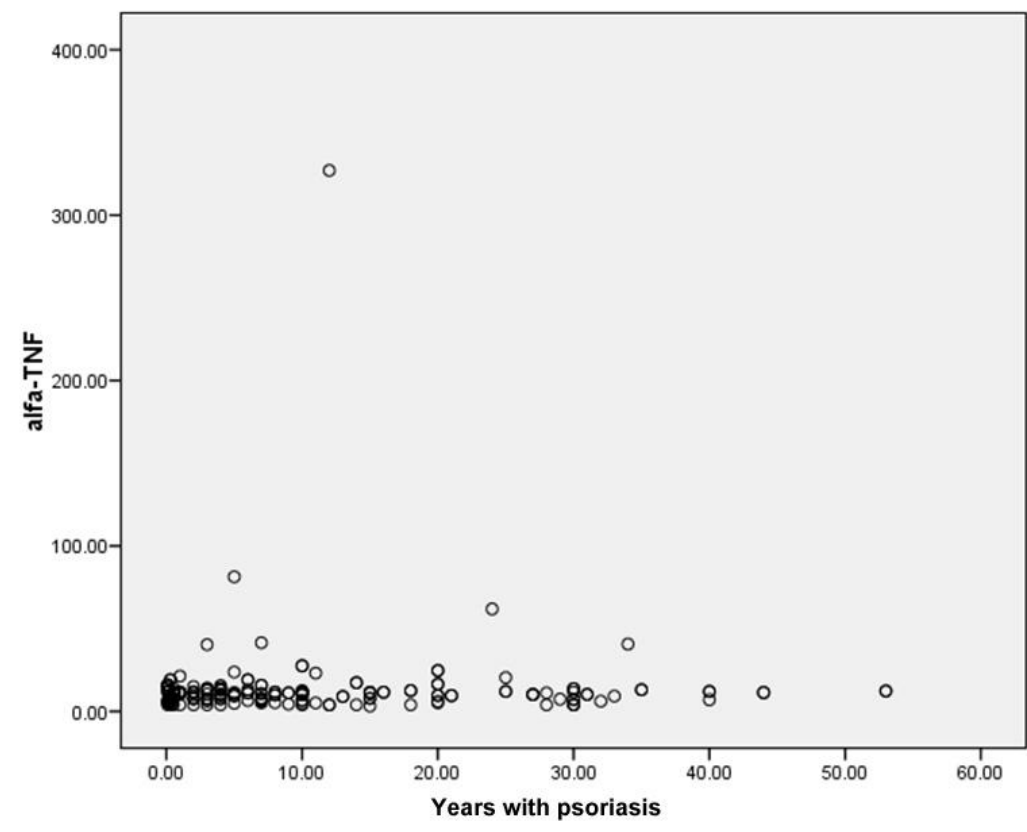

Graphic 6: correlation between alfa-TNF dhe psoriasis duration (in years)

\section{Discussion}

\section{Role of hs-CRP and TNF-alfa at patients with psoriasis}

Psoriasis is considered nowadays as an inflammatory disease similar with other immune-inflammatory disorders such as rheumatoid arthritis (RA) and lupus erithema. Similarly with these diseases, psoriasis is being labeled as an independent risk factor related in general due to the cardiovascular diseases risk increase and deaths and specifically for the myocardium infarct.

Inflammation of vessel wall is being considered to play a key role in the genesis and progress of atherosclerosis bringing up to the final moment which is the erosion or fissure and eventually the rupture of atherosclerotic plaque. 13.14

Studies on hs-CRP are showing that the latter is a micro inflammation indicator. It has turned out to be an independent risk factor for the future cardiovascular events in individuals appearing to be healthy. ${ }^{15}$

There were no differences in our study as far as it concerns in the demographic profile of the studied groups. So in other words both groups (psoriasis and the control one) were without sex and age differentiations, whose data would have brought us to the "bias information". It turned out that 99.5\% of psoriasis patients have their CRP value $>1.10 \mathrm{mg} / \mathrm{L}$, against $57.8 \%$ of those in control group, resulting in a very significant statistic change (exact fisher test:p $<0.001$ ). On the other hand, as far as it concerns the TNF-alpha values, there is a significant statistic change between psoriasis and control group.

TNF-alpha circulatory levels, as well as TNF-alpha are being noticed to increase in patients with components of metabolic syndromes such as obesity and insulin resistance. Furthermore studies show that psoriasis patients develop metabolic syndromes as a consequence of some customs or due to the impact itself psoriasis has in their life (like for instance the non healthy way of nutrition, alcohol consuming, stress, decrease of physical activity as a consequence of psoriasis symptoms),thus becoming a reason for body mass enlargement and metabolic syndromes. ${ }^{16}$

Psoriasis has a large impact in life quality by causing a high level of anxiety, depression and even suicide thoughts. The reduction of the physical and mental activity in psoriatic patients is compared with the one of an individual suffering from cancer, arthritis, heart disease, diabetes and depression. Some studies describe a key role of cytokines in pathogenesis of depression. So, a study at O'Brien et al. describes the depressive disorders being associated with the increase of production of proinflammatory cytokines, IL-6,IL-8 and TNFalfa even after the usage of humor stabilizers or antipsychotic medications. Meanwhile the treatment itself of the maniacdepressing diseases with such medications like lithium could induce psoriasis aggravation. ${ }^{17.18}$

Many other studies, where the above outcome is in conformity with them, have reported that the risk of cardiovascular diseases among patient with psoriasis is higher in patients with aggravated form of psoriasis.19, 20, 21, 22.

On the other hand it is noticed a relatively strong relation among values of PASI, alpha-TNF and hs-CRP. This input reinforces once again the idea that inflammation in psoriasis results in inflammatory cytokine increase like for instance alpha-TNF and IL-6 and with signs of a systemic inflammation such as the increase of hs-CRP levels. High CRP levels are being noticed to be related with a higher risk

Volume 4 Issue 11, November 2015 


\section{International Journal of Science and Research (IJSR) \\ ISSN (Online): 2319-7064 \\ Index Copernicus Value (2013): 6.14 | Impact Factor (2014): 5.611}

for thrombotic events. These factors seems to play a role in atherosclerosis development and in IM.

Said that, the measurement of the seric level of the abovementioned mediators, could be considered as an objective parameter of the psoriasis activity and clinical importance (PASI). These data confirm the hypothesis that psoriasis could be considered as a totally systemic disease with specific immunology mechanisms.

Nevertheless, we could not speculate in the specificity of this result since further studies must be conducted to define the role of proinflammatory cytokines in serum in pathogenesis and in their correlation with psoriasis severity.

\section{References}

[1] Gottlieb SL,Gilleaudeau P,Johnson R, et al.Response of psoriasis to a lymfocite-selective toxin(DAB389IL2)suggests a primary immune, but not keratinocite,pathogenic basis.Nat Med 1995;1(5):442-7.

[2] Piskin G,Sylva-Steenland RM,Bos JD,Teunissen MB.In vitro and in situ expression of IL-23 by keratinocytes in healthy skin and psoriasis lesions :enhanced expression in psoriatic skin .J immunol 2006;176(3):1908-15.

[3] Bos JD,Hulsebosch HJ, Krieg SR,Bakker PM,Cormane RH.Immunocompetent cells in psoriasis .In situ immunophenotyping by monoclonal antibodies. Arch Dermatol Res 1983;275(3):181-9.

[4] Griffiths CE,Powles AV,McFadden J,Baker BS, Valdimarsson H,Fry L.Long -term cyclosporine for psoriasis.Br J Dermatol 1989;120(2):253-60.

[5] Krueger G, Ellis CN.Psoriasis -recent advances in understanding its pathogenesis and treatment.J Am Acad Dermatol 2005;53:S94-S100.

[6] Chaturvedi V, Qin JZ,Denning MF,et al.Apoptosis in proliferating,senescent, and immortalized keratinocytes.J Biol Chem 1999;274:23358-67.

[7] Wei CC ,HY,Li HH,et al. IL-20; biological function and clinical implication.JBiomed Sci 2006:13:601-612.

[8] Arican O,Aral M,Sasmaz S,Ciragil P.Serum levels of TNF-alpha,IFN-Y,IL-6,IL-8,IL-12,IL-17, and IL-18 in patients with active psoriasis and correlation with disease severity.Mediators Inflamm 2005;273-279.

[9] Ikeda U,Ito T,Shimada K.Klin Cardiol 2001;24:701-704.

[10] Tillet WS, Francis T. Serological reaction in pneumonia with a non-protein somatic fraction of pneumococcus. J Exp Med;52:561-571; 1930.

[11] MacLeod CM, Avery OT. The occurrence during acute infections of a protein not normally present in the blood. II. Isolation and properties of the reactive protein. J Exp Med;73:183-191; 1943.

[12] Fredriksson T,Pettersson U.Severe psoriasis-oral terapy with a new retinoid.Dermatologica;157:238-44,1978.

[13] Piskin G,Sylva-Steenland RM,Bos JD,Teunissen MB.In vitro and in situ expression of IL-23 by keratinocytes in healthy skin and psoriasis lesions :enhanced expression in psoriatic skin .J immunol 2006;176(3):1908-15.
[14] Bos JD,Hulsebosch HJ, Krieg SR,Bakker PM,Cormane RH.Immunocompetent cells in psoriasis .In situ immunophenotyping by monoclonal antibodies. Arch Dermatol Res 1983;275(3):181-9.

[15] Ross R.Atherosclerosis -an inflammatory disease.N Engl Jn Med 1999;340:115-26.

[16] Plutzky J.Inflammatory pathways in atherosclerosis and acute coronary syndromes .Am J Cardiol 2001;88:10k$15 \mathrm{k}$.

[17] Neimann AL,Shin DB,Wang X,Margolis DJ,Troxel AB, Gelfand JM.Prevalence of cardiovascular risk factors in patients with psoriasis.J Am Acad Dermatol 2006;55(5):829-35.

[18] Complexity of the Association Between Psoriasis and Comorbidities Nijsten $\mathrm{T}$, Wakee M, J.Invest Dermatol,2009;129:1601-3. 25-28.

[19] Kimhi O,Caspi D,Bornstein NM, et al.Prevalence and risk factors $\mathrm{pf}$ atherosclerotic in patients with psoriatic arthritis.Semin Arthritis Rheum, 2007;36:203.

[20] Pasceri V,Yeh ET.A tale of two diseases:atherosclerosis and rheumatoid arthritis.Circulation ,1999;100:2124.

[21] Yeyand CM,Goronzy JJ,Liuzzo G,et al.T-cell immunity in acute coronary syndromes.Mayo Clinic Proc, 2001;76:1011.

[22] Sattar N,McCarey DW, Capell H,McInnes IB.Explaining how "high-grade" systemic inflammation accelerates vascular risk in rheumatoid arthritis.Circulation, 2003:108:2957.

Volume 4 Issue 11, November 2015 www.ijsr.net 